\title{
Modelling Revenue Management Problem under Fare Class Interval Customer Choice
}

\author{
Baohua Wang \\ Tsinghua University, Beijing, 100084, China \\ Beijing Union University, Beijing, 100025, China
}

\begin{abstract}
In order to explain customer choice conditioned on opened classes more realistically and efficiently, I assume a customer considers a discrete choice as a fare class interval and buys the lowest opened class with that interval. Unlike the traditional one-dimensioned, unstructured choice model, I model customer choice as a two-dimensioned fare class interval distribution. I take closed set as a more efficient research angle, and find out an additive principle to reduce computation. Then I turn the traditional maximized decision principle into minimized decision principle and get a new model for the maximized total revenue management problem. This model builds the foundation for more efficient explorations to revenue management problem.
\end{abstract}

Keywords-modeling; customer behavior; customer choice; operational research; revenue management

\section{INTRODUCTION}

Customer interview as well as airline information system data reveals that a customer usually buys different fare class across time. That is, a customer's buying decision is made on condition of opened set [1]. Then what drives her to buy that certain class under that opened set? As customer demand is usually a mix of priceable type and yieldable type [2], there are several fare classes in a customer's considering set [3][4], and she make eventual choice according to a certain preference order [5].

Customer choice as a permutation of fare classes is called as discrete customer choice [1]. When the permutation is a subset of the entire fare class set $f_{1}, \ldots, f_{n}$ with a few fare classes missing, the number of demand types are relatively limited. Reference [1] gave an example of choice set. Reference [6] assumed all the customers are complete priceable type [2], buying the lowest opened class. That is, the customer choice is $[n, h], h=n, \ldots, 1$. This is a particular case of FCI model, with all the customers belonging to the same layer $n$. Reference [3] took choice model as choice set (customer choice as a set of fare classes) and estimated the arriving rate of each choice set across time by maximizing likelihood function dependent of offer sets, yet they only use class set to take place individual class without looking into the structure of all the choice sets. I construct a choice model not only as a fare class set-type choice model, but also study the inner instructure of choices by clustering choice sets in to layers.

I study on a particular choice model where a customer independently makes choice in a fare class interval (FCI), and buys the lowest opened class within that FCI. For example, suppose a customer only makes choice in an FCI $[5,2]$, i.e., $\{5,4,3,2\}$. When there are trivial differences between fare products while big difference between fares [7], a customer tends to choose an FCI instead of a single fare class.

An explanation of FCI choice is customer's valuation or willing to pay (WTP) [8] on flying travel being an interval of classes rather than a single class. A customer has a lower bound of WTP because one has a lower bound flying travel utility. One has an upper bound of WTP because of her affordable budget on flying travel. All the classes among the interval are acceptable because they are all fare-affordable and utility-acceptable. Furthermore, a customer's total utility includes flying travel utility and customer surplus currency utility, i.e., a customers is price sensitive among her FCI, so although she gets more flying travel utility with higher fare class, she will eventually choose the lowest opened class among her FCI. Another rationale of FCI is anchoring effect in marketing science literature. Reference [9] found the original price of a discounted commodity had an upside-down $U$ shape effect on price expectation of customers. That is, as the discrepancy between original price and discounted price expands, customer price expectation increases at first and then decreases, which implies customers take original price at first and then take discounted price as price anchor. Applying this effect into hierarchy of fare classes, I find when valuating lower classes standing at ones upper bound of WTP, at first one takes this higher fare class as anchor, and evaluates a fare product as higher than its fare. After a breaking point, one takes the lower fare class itself as anchor, and evaluates a fare product as low as its fare. Reflected on choice behavior, one will only choose some adjacent fare classes near ones WTP upper bound.

Customer choice is traditionally explained by fare products' attributes including price. Reference [1], [4] and [10] used MNL model to determine the buying probability toward each class, by the weights of various attributes, such as customer's sensitivity on price, restrictions, among the other attributes of fare class products. The parameters of MNL model were simulated or estimated on the basis of classes [1], [5], [10]. This is a rigid model [7] because the buying probability is deterministic, independent, without buy up, and results in only one customer choice type. To differentiate the choice model, Reference [1] and [10] added a random factor, while Reference [4] shortened customer's consideration set into disjoint fare class sets. Reference [3] developed this MNL based choice model into choice sets as a hole. After preference on each class is given, a choice set will certainly contain the preference as an attribute. In this sense, FCI model is a case when customers are price sensitive within FCIs, while other attributes (such as utility) sensitive out of FCIs. Demand distribution has been modeled according to customer's valuation of the product, customer's willing to pay (WTP) [8], or customer's highest fare class choice [6]. In their models, demand distribution is 
the given proportion of a single variable on customer. My choice model is a two-dimensioned demand distribution of $[l, h]$.

My research is presented as following. In section II I construct FCI choice model. Then in section III I model the maximized total revenue problem. Section IV provides conclusion of my research.

\section{CHOICE MOdEL}

\section{A. FCI Choice Model}

Notations of FCI choice model are listed in table I.

TABLE I. NOTATIONS IN CHOICE MODEL

\begin{tabular}{ll}
\hline Terms & \multicolumn{1}{c}{ Meaning } \\
\hline$f_{j}$ & Fare class. $f_{n}<\cdots<f_{j}<\cdots<f_{1}, j=n, \ldots, 1$ \\
$I$ & Fare Class Interval. $I \equiv[l, h], l \geq h, 1 \leq l, h \leq n$ \\
$l$ & The lowest class of an FCI; Index of layers in $l$ model \\
$h$ & The highest class of an FCI; Index of layers in $h$ \\
& model \\
$d_{h}^{l}$ & Demand quantity of FCI $[l, h]$ in layer $l$ \\
$a_{l}$ & Demand quantity of layer $l$ \\
$Q\left(A_{h-1}^{l}\right)$ & Accumulative tail demand quantity of $[l, h]$ within \\
$S$ & layer $l . Q\left(A_{h-1}^{l}\right)=\sum_{k=h-1}^{1} d_{k}^{l}$ \\
$\bar{S}$ & Fare class set opened \\
$A$ & Fare class set closed \\
$A_{j}$ & Complete fare set closed \\
& Complete fare set closed with $j$ as its highest class. \\
$K$ & $A_{j} \equiv[n, j]$ \\
$q$ & Incomplete FCI. $K \equiv[l, h], l \geq h, 1 \leq l, h<n$ \\
$p$ & Closed set demand quantity (CSQ) \\
\hline
\end{tabular}

I study a context where there is only one single leg of one airline company, who provide $n$ class fare products $f_{1}, \ldots, f_{n}$, with total capacity $C$. A customer type is defined as a FCI $\equiv[l, h], l \geq h, 1 \leq l \leq n$, where $l, h$ is separately the lowest and highest fare class the customer chooses. I assume the customer eventually buys the lowest opened class within her FCI, and customer types are independent with each other, thereby the capacity control problem transits from being based on fare classes to being based on fare class sets. Furthermore, I assume that customer demand follows a deterministic discrete distribution denoted by $F\left(d_{h}^{l}\right)$, where $d_{h}^{l}$ is the demand of FCI $[l, h], l \geq h, 1 \stackrel{d^{h}}{-}$ $l \leq n . d_{h}^{l}$ could be directly investigated from customers. $l$ is taken as fare class layer, and $[l, h]$ is taken as the FCI within layer $l$. Then $a_{l}=\sum_{h=1}^{l} d_{h}^{l}, a_{l} \in[0,1]$ is the layer demand, and $Q\left(A_{h}^{l}\right)=\sum_{k=h}^{1} d_{k}^{l} / a_{l}, h=1, \ldots, l, l=1, \ldots, n$ is the cumulative demand of interval $[h, 1]$ within layer $l$. Figure I illustrates the distribution of customer demand under FCI model.

Each of the four curves in figure I separately denotes the distribution densities of the choice intervals with the lower bound, i.e., the layer of $f_{n}, \ldots, f_{1}$. Each layer is de-

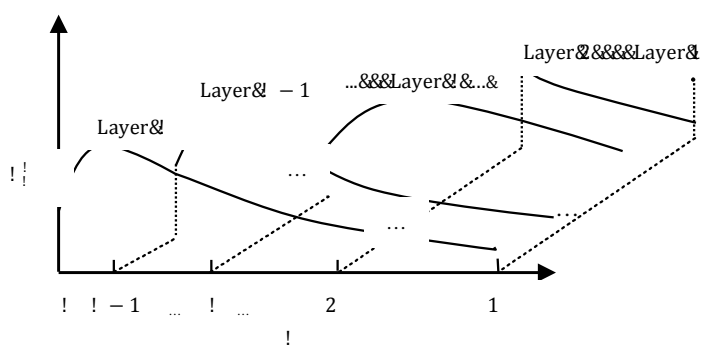

FIGURE I. FCI CHOICE MODEL picted as a line, and a point $h$ in a curve $l$ represents the demand proportion of FCI $[l, h]$ within layer $l$, whose value is $d_{h}^{l} / a_{l}$.

Let's take example 1 to illustrate this model. Referring to the fare structure of the Beijing - Shanghai leg, China Airline on www.ctrip.com, I get six fare classes: 520, 650, $750,1200,2100,3800$. Setting up $d_{h}^{l}$ properly I get a FNAPO case, as seen in table II. Table II also list $Q\left(A_{h}^{l}\right)$, which is computed according to $d_{h}^{l}$.

TABLE II. THE INTERVAL DEMAND OF EXAMPLE 1

\begin{tabular}{llllll}
\hline \multicolumn{1}{c}{$\boldsymbol{l}, \boldsymbol{h}]$} & $\boldsymbol{d}_{\boldsymbol{h}}^{\boldsymbol{l}}$ & $\boldsymbol{Q}\left(\boldsymbol{A}_{\boldsymbol{h}}^{\boldsymbol{l}}\right)$ & \multicolumn{1}{c}{$[\boldsymbol{l}, \boldsymbol{h}]$} & $\boldsymbol{d}_{\boldsymbol{h}}^{\boldsymbol{l}}$ & $\boldsymbol{Q}\left(\boldsymbol{A}_{\boldsymbol{h}}^{\boldsymbol{l}}\right)$ \\
\hline$[1]$ & 0.0200 & 1.00 & {$[5,4]$} & 0.0950 & 0.68 \\
\hline$[2]$ & 0.0464 & 1.00 & {$[5,4,3]$} & 0.0300 & 0.30 \\
{$[2,1]$} & 0.0336 & 0.42 & {$[5,4,3,2]$} & 0.0250 & 0.18 \\
\hline$[3]$ & 0.0975 & 1.00 & {$[5,4,3,2,1]$} & 0.0200 & 0.08 \\
{$[3,2]$} & 0.0300 & 0.35 & {$[6]$} & 0.1317 & 1.00 \\
{$[3,2,1]$} & 0.0225 & 0.15 & {$[6,5]$} & 0.0540 & 0.56 \\
\hline$[4]$ & 0.1440 & 1.00 & {$[6,5,4]$} & 0.0600 & 0.38 \\
{$[4,3]$} & 0.0440 & 0.28 & {$[6,5,4,3]$} & 0.0300 & 0.18 \\
{$[4,3,2]$} & 0.0080 & 0.06 & {$[6,5,4,3,2]$} & 0.0150 & 0.08 \\
{$[4,3,2,1]$} & 0.0040 & 0.02 & {$[6,5,4,3,2,1]$} & 0.0093 & 0.03 \\
\hline$[5]$ & 0.0800 & 1.00 & & & \\
\hline
\end{tabular}

According to $d_{h}^{l}$ of table I, I get the accumulative demand distribution of example 1 , shown in figure II.

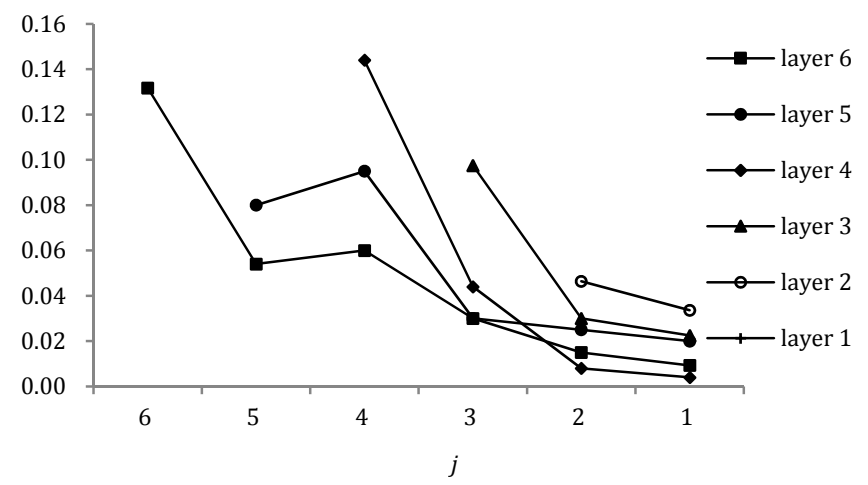

FIGURE II. FCI DEMAND DISTRIBUTION OF EXAMPLE 1

\section{B. Closed Set Research Angle}

No let's find out which view angle is the simplest in analysis and computation.

When an arbitrary FCI $[l, h]$ is closed, the choice of customers in layer $k, k \in[l, h]$ shifts from class $k$ to class $h-1$. Meanwhile, customers who choose higher classes $h-1, \ldots, 1$ have no demand in class $k$, and customers who choose lower classes $n, \ldots, l+1$ still buy classes lower than $k$. So only the choice of customers in layer $k, k \in$ $[l, h]$ is affected. For the same rationale, I analyze the changes in demand of various classes, concluded in Table III. 
TABLE III. DEMAND CHANGES UNDER CLOSED / OPENED FCI

\begin{tabular}{|c|c|c|}
\hline Class & Under closed FCI & Under opened FCI \\
\hline$k, k \in[l, h]$ & $\begin{array}{l}\text { From } k \text { to } h-1 \\
\text { within layer } l\end{array}$ & $\begin{array}{l}\text { From } 0 \text { to } k \text { within } \\
\text { layer } l\end{array}$ \\
\hline$h-1, \ldots, 1$ & No demand in $k$ & No demand in $k$ \\
\hline $\begin{array}{l}m, m \\
\in[n, l+1]\end{array}$ & Still $m$ & $\begin{array}{l}\text { Still } m \text {, or }{ }^{\mathrm{a}} \text { from } 0 \\
\text { to } k\end{array}$ \\
\hline
\end{tabular}

From table III we can see, the simplest scenario is to study closed sets. The simplification on computing isolated sets when they are closed is a sense of constructing FCI model. From now on, all the sets or classes in remained sections are referred to closed ones if no particular statements are offered.

\section{Computations on Closed Set and Additive Principle}

According to FCI model, the decreased demand quantity brought by layer $k$, defined as layer closed set quantity (layer CSQ) is $q_{k}(I)=a_{k}\left(1-Q\left(A_{h-1}^{k}\right)\right)$, while the decreased revenue, defined as layer closed set revenue (layer CSR) brought by layer $k$ is $r_{k}(I)=a_{k}\left(p_{k}-\right.$ $\left.p_{h-1} Q\left(A_{h-1}^{k}\right)\right)$. The FCI's total CSQ to the leg, defined as CSR, is $q(I)=\sum_{k=l}^{h}\left(a_{k}\left(1-Q\left(A_{h-1}^{k}\right)\right)\right.$, the FCI's total CSR to the leg, defined as CSR, is $r(I)=\sum_{k=l}^{h} a_{k}\left(p_{k}-\right.$ $\left.p_{h-1} Q\left(A_{h-1}^{k}\right)\right)$, and the FCI's closed set price (CSP) to the leg is $p(I) \equiv \frac{r(\{l, h\})}{q(\{l, h\})}=\frac{\sum_{k=l}^{h} a_{k}\left(p_{k}-p_{h-1} Q\left(A_{h-1}^{k}\right)\right)}{\sum_{k=l}^{h} a_{k}\left(1-Q\left(A_{h-1}^{k}\right)\right)}$. For the same rationale, non-adjacent FCIs will also not affect the demand of each other. To summarize we have additive principle as stated in property 1 .

Property 1. For $\forall I=[l, h]$ and for $\forall \bar{S}, \bar{S}=\cup I(\bar{S})$, where $I(\bar{S})$ s are the non-adjacent FCIs composing $\bar{S}$, there exists additive principle as $q(I)=\sum q_{k}(I), r(I)=\sum r_{k}(I)$, $q(\bar{S})=\sum q(I(\bar{S})), r(\bar{S})=\sum r(I(\bar{S})$.

Table IV lists the additive principle separately at the level of layer, FCI and $\bar{S}$. Note that layer CSQ is the demand quantity at class level [1].

Table IV implies that under FCI model, there exists additive principle from class to FCI and through to general set. It facilities the computation and decision directly on sets instead of on classes.

TABLE IV. ADDITIVE PRINCIPLE ABOUT FCI AND ARBITRARY SET

\begin{tabular}{ccc}
\hline CSP & CSR, CSQ & $\begin{array}{c}\text { Layer CSR, CSQ to Layer } \\
\boldsymbol{k}, \boldsymbol{k}=\boldsymbol{l}, \ldots, \boldsymbol{h}\end{array}$ \\
\hline$p(I)=\frac{r(I)}{q(I)}$ & $r(I)=\sum_{k=h}^{l} r_{k}(I)$ & $\begin{array}{l}r_{k}(I) \\
=a_{k}\left(f_{k}-f_{h-1} Q\left(A_{h-1}^{k}\right)\right)\end{array}$ \\
$q(I)=\sum_{k=h}^{l} q_{k}(I)$ & $q_{k}(I)=a_{k}\left(1-Q\left(A_{h-1}^{k}\right)\right)$ \\
$p(\bar{S})=\frac{r(\bar{S})}{q(\bar{S})}$ & $r(\bar{S})=\sum^{2} r(I(\bar{S}))$ \\
\end{tabular}

\section{MAXIMIZED REVENUE MODEL}

\section{A. Traditional Model}

I construct maximized total revenue model based on the Bellman equation in Reference [1]. Notations of maximized total revenue model are included in table $\mathrm{V}$.

TABLE V. NOTATIONS IN MAXIMIZED TOTAL REVENUE MODEL

\begin{tabular}{ll}
\hline Terms & \multicolumn{1}{c}{ Meaning } \\
\hline$\lambda$ & Customer arriving rate within one stage. $\lambda \leq 1$ \\
$t$ & Index of remaining time before departure \\
$x$ & Index of remaining seat on the airline \\
$R$ & Revenue gained when class set $S$ is opened \\
$Q$ & Demand probability when class set $S$ is opened \\
$V_{t}(x)$ & Total revenue gained by sailing $x$ units of seats \\
$\Delta V_{t}(t, x)$ & Revenue gained by sailing the $x$ th seat at time $t$ \\
$v$ & $\Delta V_{t}(t, x) \equiv V_{t}(x-1)-V_{t}(x)$ \\
$\bar{S}^{*}$ & Bid price of the $x$ th seat at time $t . v \equiv \Delta V_{t}(t, x)$ \\
\hline
\end{tabular}

When customer choice structure is known, and customer arriving rate is $\lambda$, the Bellman equation is

$$
V_{t}(x)=\max \{\lambda(R(S)-v Q(S))\}+V_{t-1}(x)
$$

where bid price $v \equiv V_{t-1}(x)-V_{t-1}(x-1)$ denotes the marginal revenue gained by selling the $x$ st capacity at stage $t-1, v Q(S)$ means the revenue gained by selling $Q(S)$ units of the opening set remains $S^{*}(t-1, x)$, and $R(S)-$ $v Q(S)$ means the incremental revenue under bid price $v$ yielded by opening set $S$ compared with the revenue yielded by last stage opening set $S^{*}(t-1, x)$. Decision principle $\max \{\lambda(R(S)-v Q(S))\}$ is to find $S^{*}(t, x)$ with the highest incremental revenue at stage $t$. Let $S_{0}$ denote the last opened set, then $R(S)-v Q(S)$ represents the incremental revenue gained by opening $S$ than by opening $S_{0}$. Furthermore, the value of $v$ is monotonically decreasing with remained time $t$ and remained capacity $x$.

\section{B. New Decision Principle}

From the Bellman equation we can see that the decision principle of the problem is $\max \{R(S)-v Q(S)\}$. In order to simplify computation, I transform it into that under closed set research angle.

THEOREM 1. Under FCI model, decision principle $\max \{R(S)-v Q(S)\}$ is equivalent to $\min \{r(\bar{S})+v(1-$ $q(\bar{S})\}$.

$$
\text { ProOF. First verify } R\left(A_{n}\right)=R(S)+q(\bar{S}) \text {. }
$$

There are two contexts with the queue of closed sets and opened sets. That is, to begin with a closed set or with an opened set. See as in figure III and figure IV, where blanks denote closed sets, while shadows denote opened sets. The symbols only denote closed sets.
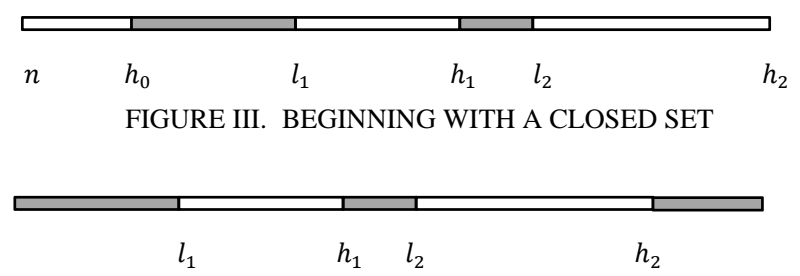

FIGURE IV. BEGINNING WITH AN OPENED SET 
When beginning with a closed set, $A_{h_{0}}=\left[n, h_{0}\right], K_{i}=$ $\left[l_{i}, h_{i}\right], i=1, \ldots, N$, we have $\bar{S}=\left[n, h_{0}\right] \cup\left(\cup_{i=1}^{N}\left[l_{i}, h_{i}\right]\right)$, $S=\bigcup_{i=0}^{N-1}\left[h_{i}-1, l_{i+1}+1\right]$. According to additive principle,

$$
\begin{gathered}
r(\bar{S})=r\left(A_{h_{0}}\right)+\sum_{i=1}^{N} r\left(K\left(\left[l_{i}, h_{i}\right]\right)\right)=\sum_{k=n}^{h_{0}} a_{k}\left(f_{k}-\right. \\
\left.f_{h_{0}-1} Q\left(A_{h_{0}-1}^{k}\right)\right)+\sum_{i=1}^{N} \sum_{k=l_{i}}^{h_{i}} a_{k}\left(f_{k}-f_{h_{i}-1} Q\left(A_{h_{i}-1}^{k}\right)\right), \\
R(S)=\sum_{i=0}^{N} R\left(K\left(\left[h_{i}-1, l_{i+1}+1\right]\right)\right)= \\
\sum_{i=0}^{N}\left(\sum_{k=h_{i}-1}^{l_{i+1}+1} a_{k} f_{k}+\sum_{k=l_{i}}^{h_{i}} a_{k} f_{h_{i}-1} Q\left(A_{h_{i}-1}^{k}\right)\right) .
\end{gathered}
$$

Hence $\quad r(\bar{S})+R(S)=\sum_{k=n}^{h_{0}} a_{k} f_{k}+\sum_{k=h_{0}-1}^{l_{1}+1} a_{k} f_{k}+$ $\sum_{i=1}^{N}\left(\sum_{k=l_{i}}^{h_{i}} a_{k} f_{k}+\sum_{k=h_{i}-1}^{l_{i+1}+1} a_{k} f_{k}\right)=\sum_{k=n}^{1} a_{k} f_{k} \quad$. Since $R\left(A_{n}\right)=\sum_{k=n}^{1} a_{k} f_{k}$, we have $r(\bar{S})+R(S)=R\left(A_{n}\right)$.

When beginning with an opened set, $A_{l_{1}}=\left[n, l_{1}+1\right]$, closed set $K_{i}=\left[l_{i}, h_{i}\right], i=1, \ldots, N$, we have $\bar{S}=$ $\left(\cup_{i=0}^{N}\left[l_{i}, h_{i}\right]\right), S=\left[n, l_{0}+1\right] \cup_{i=1}^{N}\left[h_{i}-1, l_{i+1}+1\right]$. By the same rationale as in the context of beginning with a closed set, I also have $r(\bar{S})+R(S)=R\left(A_{n}\right)$.

Then, by the same rationale as that with $r(\bar{S})+R(S)=$ $R\left(A_{n}\right)$, we have $q(\bar{S})+Q(S)=Q\left(A_{n}\right)$.

As $Q\left(A_{n}\right)=\sum_{k=n}^{1} a_{k}=1$, we have $q(\bar{S})+Q(S)=1$. Thus under any contexts, I always have $Q(S)=Q\left(A_{n}\right)$ $q(\bar{S})=1-q(\bar{S})$.

Therefore, $\quad R(S)-v Q(S)=\left(R\left(A_{n}\right)-r(\bar{S})\right)-v(1-$ $q(\bar{S}))=R\left(A_{n}\right)-(r(\bar{S})+v(1-q(\bar{S}))$.

Since $R\left(A_{n}\right)$ is the same for all the $\bar{S}, \max \{R(S)-$ $v Q(S)\}$ is equivalent to $\min \{r(\bar{S})+v(1-q(\bar{S})\}$.

\section{New Model}

Accoring to problem (1), theorem 1, and the definition of bid price $v$, the maximized revenue model is transformed to

$$
v(t, x)=\lambda R\left(A_{n}\right)-\lambda \min \{r(\bar{S})+v(1-q(\bar{S})\}
$$

Problem (2) contains a dynamic programming (DP) game. In each stage $t$, airline company acquires $v(t, x), x=1, \ldots, C$ and chooses $\bar{S}^{*}(t, x)$. After that, each customer buys a fare class according to $\bar{S}^{*}(t, x)$ and her FCI. Behavior of all the customers generate $v(t+$ $1, x), x=1, \ldots, C$, which will trigger the game of the next stage $t+1$. As $v$ is monotonically decreasing, one or more optimal policies $\left(v_{i}, \bar{S}_{i}^{*}\right), i=1, \ldots$ will be found.

\section{CONCLUSiOn}

In this research I construct a new customer choice model -FCI model, where an FCI $[l, h]$ is composed of several adjacent fare classes. This model reflects the fact that a sami-price sensitive customer usually chooses among several fare classes and eventually buy the lowest opened fare class. I find under FCI model, computation is simplified under closed set research angle rather than under traditional opened set research angle. So a new decision cretiria is developed and a new maximized total revenue model is formed. The maximized model reflects the game between the airline company and its customers, and airline's optimal policy will be formed during the game process. This modeling process builds the foundation for further researches such as analysis on optimal policy, total revenue, buy up behavior and its impact under FCI choice model.

\section{REFERENCES}

[1] K. Talluri and G. van Ryzin, "Revenue management under a general discrete choice model of consumer behavior,” Management Science, vol. 50(1), pp. 15-33, 2004.

[2] A. Boyd, R. Kallesen, "The science of revenue management when passengers purchase the lowest available fare,” Journal of Revenue and Pricing Management, vol. 3(2), pp. 171-177, 2004.

[3] W. Haensel and G. Koole, "Estimating unconstrained demand rate functions using customer choice sets," Journal of Revenue and Pricing Management, vol. 10(5), pp. 438-454, 2010.

[4] Q. Liu and G. van Ryzin, “On the choice-based linear programming model for network revenue management,” Manufacturing and Service Operations Management, vol. 10(2), pp. 288-310, 2008.

[5] G. Vulcano, G. van Ryzin and W. Chaar, "Choice-based revenue management: an empirical study of estimation and optimization," Manufacturing and Service Operations Management, vol. 12(3), pp. 371-392, 2010.

[6] T. Fiig, K. Isler, C. Hopperstad, and P. Belobaba, “Optimization of mixed fare structures: theory and applications,” Journal of Revenue and Pricing Management, vol. 9(1/2), pp. 152-170, 2010.

[7] L. Williamson, “Airline network seat inventory control: Methodologies and revenue impacts,” Ph.D. thesis, Massachusetts Institute of Technology, Cambridge, MA, 1992.

[8] X. Su, "Intertemporal pricing with strategic customer behavior," Management Science, vol. 53(5), pp. 726-741, 2007.

[9] P. K. Kopalle, J. Lindsey-Mullikin, “The impact of external reference price on consumer price expectations,” Journal of Retailing, vol. 79, pp. 225-236, 2003.

[10] G. Gallego, R. Ratliff and S. Shebalov, “A general attraction model and sales-based linear program for network revenue management under customer choice,” Operations Research, vol. 63(1), pp. 212-232, 2015 . 